\title{
Pregabalin as a probable cause of central serous chorioretinopathy: Two case reports
}

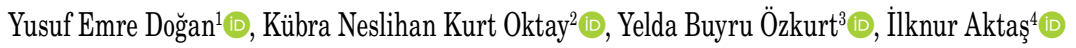 \\ ${ }^{1}$ Department of Ophthalmology, Dünya Göz Hospitals Group, Istanbul, Turkey \\ ${ }^{2}$ Department of Physical Medicine and Rehabilitation, Yeditepe University Hospital, Istanbul, Turkey \\ ${ }^{3}$ Department of Ophthalmology, University of Health Sciences Fatih Sultan Mehmet Training and Research Hospital, Istanbul, Turkey \\ ${ }^{4}$ Department of Physical Medicine and Rehabilitation, University of Health Sciences, Fatih Sultan Mehmet Training and Research Hospital, Istanbul, Turkey
}

Received: October 19, 2019 Accepted: February 04, 2020 Published online: December 01, 2021

\begin{abstract}
Pregabalin is commonly used for the treatment of neuropathic pain and is attributed to adverse effects of peripheral vasodilation and peripheral edema. Central serous chorioretinopathy (CSCR) is characterized by choroidal fluid leaks from choroidal vessels under the retina, causing focal retinal detachment with macular vision loss. Herein, we report two cases admitted to our clinic with vision loss while under pregabalin treatment. Upon eye examination, both patients were diagnosed with acute CSCR. Pregabalin treatment was discontinued upon the diagnosis of CSCR. We consider that the use of pregabalin in the presented two cases may be the causal effect of the CSCR diagnosis, as pathophysiology of CSCR is in parallel with the edema-related adverse effects of pregabalin.
\end{abstract}

Keywords: Adverse drug reaction, central serous chorioretinopathy, pregabalin.

Central serous chorioretinopathy (CSCR) is a chorioretinal disease which usually affects the macula and is characterized by serous retinal detachment, often associated with focal pigment epithelial detachments, choroidal hyperpermeability, and increased choroidal thickness. Risk factors for CSCR are male sex, higher education level, alcohol exposure, smoking, hypertension, coronary heart disease, obstructive sleep apnea, autoimmune diseases, Helicobacter pylori infection, type-A personality, stress disorder, steroid treatment, pregnancy, and hypermetropia. ${ }^{[1]}$ Central serous chorioretinopathy can be classified as acute or chronic (longer than three or six months).$^{[1]}$ Despite the improvements in imaging techniques and numerous studies, pathophysiology of CSCR has not been clearly elucidated, yet. The main pathologies which can cause CSCR are impaired choroidal vascular autoregulation induced by steroids, catecholamines or sympathomimetic agents, and loss of retinal pigment epithelial barrier function. ${ }^{[2]}$

Pregabalin, a gamma-aminobutyricacid (GABA) analog, relieves neuropathic pain by suppressing the secretion of pain-related mediators in the synapses through binding to $\alpha 2 \delta$ subunits of $\mathrm{Ca}^{+}$channels in the central nervous system. ${ }^{[3]}$ The United States Food and Drug Administration (FDA) approved pregabalin's clinical use for the treatment of postherpetic neuralgia, fibromyalgia, diabetic peripheral neuropathic pain, and several other neuropathic pains. Dizziness, vertigo, incoordination, balance disorder, ataxia, diplopia, blurred vision, amblyopia, tremor, somnolence, confusional state, disturbance, inattention, thinking abnormally, euphoria, asthenia, fatigue, edema, peripheral edema, ${ }^{[4,5]}$ dry mouth, and constipation are

Corresponding author: Kübra Neslihan Kurt Oktay, MD. Yeditepe Üniversitesi Hastanesi, Fiziksel Tip ve Rehabilitasyon Kliniği, 34718 Ataşehir, İstanbul, Türkiye. 
the reported common adverse effects of pregabalin. ${ }^{[6-9]}$ Possible heart failure exacerbation associated with pregabalin is also reported in a case report. ${ }^{[10]}$ The most common ocular side effects of pregabalin are blurred vision, abnormal vision, conjunctivitis, and diplopia, while, less frequently, ocular pain, ocular swelling, photosensitivity reaction, dry eye, blepharitis, strabismus, keratitis, uveitis, photopsia, and irritation are reported. ${ }^{[7,11]}$ Pregabalin overdose may lead to bilateral vision loss due to bilateral serous macular detachment as reported by a single suicide attempt. ${ }^{[11]}$ Another case report suggests that topiramate, an antiepileptic agent, may be linked to acquired myopia followed by neurosensory retinal detachment at the posterior pole in left eye. ${ }^{[12]}$ Pregabalin add-on therapy has been also reported as a probable cause for the occurrence of downbeat nystagmus. $^{[13]}$

To the best of our knowledge, there are no case reports in the literature showing the occurrence of CSCR with pregabalin treatment. Herein, we present two cases of CSCR while under pregabalin treatment for neuropathic pain.

\section{CASE REPORT}

Case 1- A 63-year-old female patient presented to our clinic with a history of three months of decrease in central vision in her right eye (oculus dextrus [OD]). She had no medical history of steroid use and she was non-smoker. She had a history of cervical spine surgery for disc herniation 11 months earlier to her admission to our clinic. Due to her cervical radiculopathy symptoms, she was given pregabalin $300 \mathrm{mg} /$ day $(2 \times 150 \mathrm{mg})$ for nine months by her physiatrist. She was neither on any other medication nor had any other illnesses. She had no history of ocular disease. Upon eye examination, the best corrected visual acuity (BCVA) was 20/30 and $20 / 20$ in OD and left eye (oculus sinister [OS]) respectively. Slit-lamp examination findings and eye pressures were normal for both eyes. Fundus examination revealed oval serous retinal detachment in OD. Optical coherence tomography (OCT) showed subretinal fluid at the macula (Figure 1a). Furthermore, fluorescein angiography (FA) demonstrated classic ink-blot pattern with expansile hyperfluorescence (Figure 1b-c).
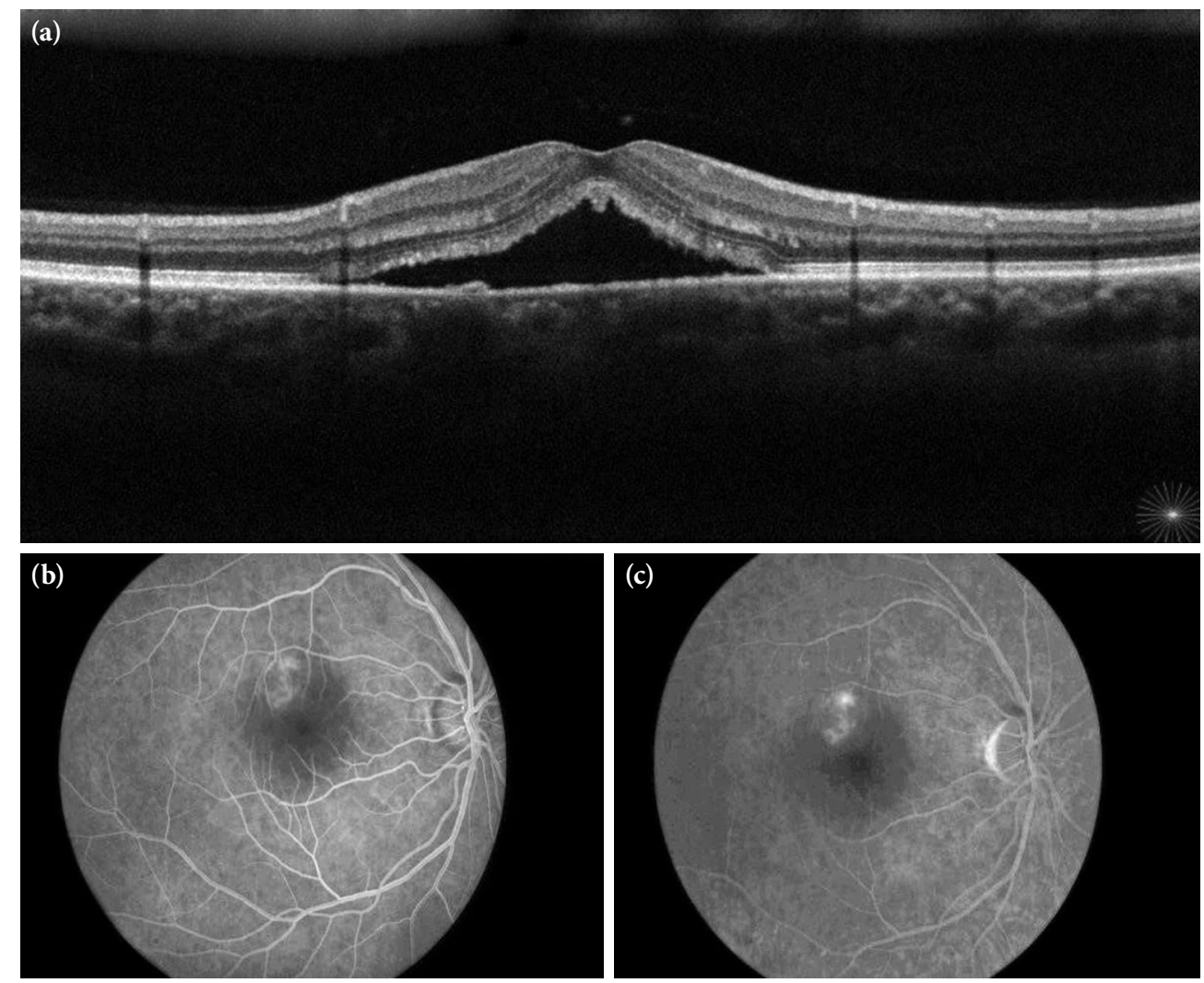

Figure 1. Diagnostic imaging findings of the first case. (a) Optical coherence tomography showing subretinal fluid in macula. (b) Fluorescein angiography showing early hyperfluorescence and (c) expansion of hyperfluorescence. 

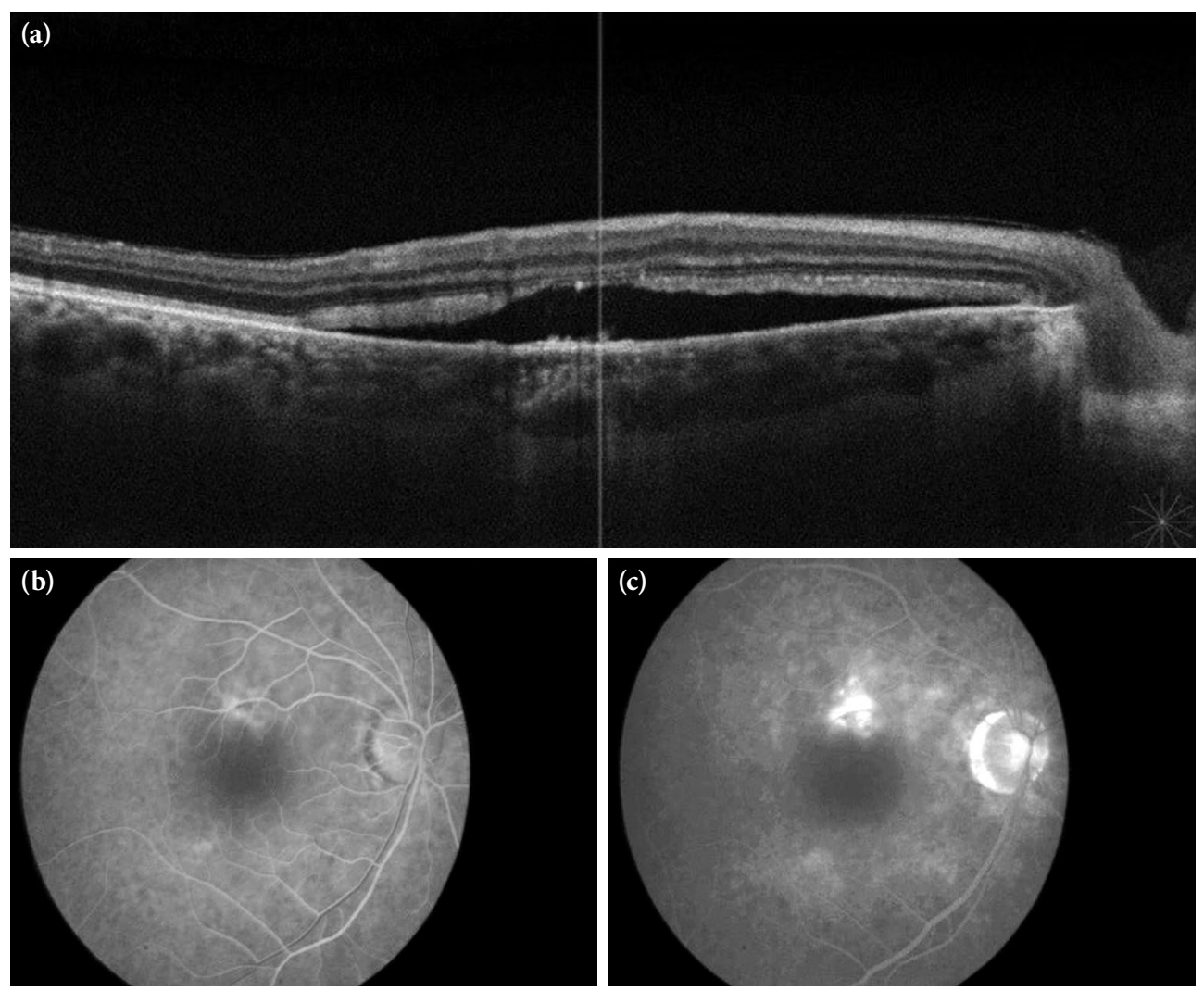

Figure 2. Diagnostic imaging findings of the second case. (a) Optical coherence tomography showing large subretinal fluid extending beyond macula. (b) Fluorescein angiography showing early hyperfluorescence superior to fovea and (c) expansion of hyperfluorescence.

Pregabalin treatment was discontinued. The patient was informed about a probable pregabalin-induced CSCR and started to be followed-up by our departments. Written informed consent was obtained from the patient for publication of this case report.

Case 2- A 45-year-old male patient was admitted to clinic with a history of three months of vision loss and metamorphopsia in his left eye. He had no history of ocular disease. He had a medical history of diabetes mellitus and chronic musculoskeletal pain; his current medication included metformin $1,000 \mathrm{mg} /$ day, acetylsalicylic acid $100 \mathrm{mg} /$ day, and pregabalin $225 \mathrm{mg}$ twice a day that he was taking for seven months. The patient was non-smoker, had no other known illnesses, and was not on any other medication that could be associated with CSCR. Upon eye examination, the BCVA values were 20/20 and 20/50 in OD and OS, respectively. Anterior segment examination findings and ocular pressures were normal for both eyes. Fundus examination revealed oval serous retinal detachment in the OS.
Further OCT and FA examinations revealed large subretinal fluid (Figure 2a), and ink-blot pattern with expansile hyperfluorescence, respectively (Figure 2b, c). Both findings were consistent with a typical CSCR diagnosis. Thereupon, pregabalin treatment was discontinued and the patient was informed about a probable pregabalin-induced CSCR diagnosis. The patient is started to be followed-up by our departments and written informed consent was obtained from the patient for publication of this case report.

\section{DISCUSSION}

To the best of our knowledge, this is the first observational case report potentially linking CSCR to the use of pregabalin. We hypothesize that pregabalin use in these two patients with vision loss could be the causal effect of their CSCR diagnosis. Although these cases could be coincidental findings with a small dataset and, therefore, further researches are needed to prove our hypothesis, the mechanism of action of pregabalin and its potential side effects 
are in parallel with the typical symptoms of CSCR. Specifically, pregabalin as an L-type calcium channel antagonist can lead to vasodilation of choroidal vessels in blood-retinal barrier which, in turn, can cause edema in retina, similar to the well-known peripheral edema caused by pregabalin and other calcium channel blockers during hypertension treatment. ${ }^{[8,10,14]}$ Interestingly, case reports in the literature suggest that pregabalin overdose may lead to macular bilateral vision loss due to bilateral serous macular detachment. ${ }^{[1]}$ Topiramate, another antiepileptic agent, can also cause neurosensory retinal detachment. ${ }^{[12]}$ Furthermore, pregabalin add-on therapy may result in downbeat nystagmus. ${ }^{13}$ These case reports are in parallel indicating a probable causal link between antiepileptic medications and occurrence of ocular disorders such as CSCR.

In conclusion, based on our observations, we updated our clinical protocols to accommodate a pre-ophthalmological check-up for patients under pregabalin treatment. The patients presented in this study are still under follow-up with a fundus examination and OCT six months after the diagnosis, since observation is the initial standard protocol for patients with acute CSCR, which usually resolves spontaneously without an intervention, and further treatment is only needed upon recurrence or development of chronicity. ${ }^{[15-18]}$ We plan to continue gathering new data from new patients under pregabalin treatment and report our findings with a larger dataset. Nonetheless, further prospective, observational studies are needed to determine the potential causative relationship between pregabalin and CSCR.

\section{Declaration of conflicting interests}

The authors declared no conflicts of interest with respect to the authorship and/or publication of this article.

\section{Funding}

The authors received no financial support for the research and/or authorship of this article.

\section{REFERENCES}

1. Wong KH, Lau KP, Chhablani J, Tao Y, Li Q, Wong IY. Central serous chorioretinopathy: what we have learnt so far. Acta Ophthalmol 2016;94:321-5.

2. Daruich A, Matet A, Dirani A, Bousquet E, Zhao M, Farman N, et al. Central serous chorioretinopathy: Recent findings and new physiopathology hypothesis. Prog Retin Eye Res 2015;48:82-118.
3. Wustmann T, Piro J, Gutmann P. Metabolic considerations in a case of pregabalin-induced edema. Pharmacopsychiatry 2009;42:75-6.

4. Gallagher R, Apostle N. Peripheral edema with pregabalin. CMAJ 2013;185:E506.

5. Guzelkucuk U, Duman I, Yilmaz B, Tan AK. Reversible post-pregabalin peripheral edema in a spinal cord injury patient. Spinal Cord 2012;50:472-3.

6. Zaccara G, Gangemi P, Perucca P, Specchio L. The adverse event profile of pregabalin: a systematic review and meta-analysis of randomized controlled trials. Epilepsia 2011;52:826-36.

7. Lyrica $^{\oplus}$ (pregabalin) Prescribing Information. Pfizer Pharmaceuticals LLC. New York, NY 10017. June 2012. Avaelable at: http://labeling.pfizer.com/showlabeling. aspx?id=561. [Accessed: September 14, 2019]

8. Toth C. Drug safety evaluation of pregabalin. Expert Opin Drug Saf 2012;11:487-502.

9. Semel D, Murphy TK, Zlateva G, Cheung R, Emir B. Evaluation of the safety and efficacy of pregabalin in older patients with neuropathic pain: results from a pooled analysis of 11 clinical studies. BMC Fam Pract 2010;11:85.

10. Page RL 2nd, Cantu M, Lindenfeld J, Hergott LJ, Lowes BD. Possible heart failure exacerbation associated with pregabalin: case discussion and literature review. J Cardiovasc Med (Hagerstown) 2008;9:922-5.

11. Tanyıldız B, Kandemir B, Mangan MS, Tangılntız A, Göktaş E, Şimşek Ş. Bilateral Serous Macular Detachment After Attempted Suicide with Pregabalin. Turk J Ophthalmol 2018;48:254-7.

12. Mazumdar S, Tripathy K, Sarma B, Agarwal N. Acquired myopia followed by acquired hyperopia due to serous neurosensory retinal detachment following topiramate intake. Eur J Ophthalmol 2019;29:NP21-NP24.

13. Ekinci AS, Ciftci S, Cavus B, Aydogdu I. Could pregabalin cause oculomotor symptoms in lower dose? A case with down beat nystagmus as a side effect. Acta Neurol Belg 2017;117:777-8.

14. Makani H, Bangalore S, Romero J, Htyte N, Berrios RS, Makwana $\mathrm{H}$, et al. Peripheral edema associated with calcium channel blockers: incidence and withdrawal rate--a meta-analysis of randomized trials. J Hypertens 2011;29:1270-80.

15. Nicholson B, Noble J, Forooghian F, Meyerle C. Central serous chorioretinopathy: update on pathophysiology and treatment. Surv Ophthalmol 2013;58:103-26.

16. Semeraro F, Morescalchi F, Russo A, Gambicorti E, Pilotto A, Parmeggiani F, et al. Central serous chorioretinopathy: Pathogenesis and management. Clin Ophthalmol 2019;13:2341-52.

17. Gülkaş S, Şahin Ö. Current Therapeutic Approaches to Chronic Central Serous Chorioretinopathy. Turk J Ophthalmol 2019;49:30-9.

18. Hanumunthadu D, Tan ACS, Singh SR, Sahu NK, Chhablani J. Management of chronic central serous chorioretinopathy. Indian J Ophthalmol 2018;66:1704-14. 